\title{
Giant magnetoresistance of magnetic multilayer point contacts
}

\author{
K. Wellock, S. J. C. H. Theeuwen, J. Caro, N. N. Gribov,* R. P. van Gorkom, \\ and S. Radelaar \\ Delft Institute of Microelectronics and Submicron Technology (DIMES), Delft University of Technology, Lorentzweg 1, \\ 2628 CJ Delft, The Netherlands \\ F. D. Tichelaar \\ National Centre for HREM, Laboratory of Material Science, Delft University of Technology, Rotterdamseweg 137, \\ 2628 AL Delft, The Netherlands \\ B. J. Hickey and C. H. Marrows \\ Department of Physics and Astronomy, EC Stoner Laboratory, University of Leeds, Leeds LS2 9JT, United Kingdom
}

(Received 15 January 1999; revised manuscript received 11 June 1999)

\begin{abstract}
We have studied the giant magnetoresistance (GMR) in magnetic multilayer point contacts of three different types. The first generation contacts were made by deposition with molecular-beam epitaxy (MBE) of an uncoupled $\mathrm{Co} / \mathrm{Cu}$ multilayer on a pre-etched hole in a thin membrane. These devices exhibited a GMR, but its ratio was low and, as deduced from finite element calculations, in many cases was dominated by the resistance of the multilayer electrode. When corrected for this, the maximum point-contact GMR was $3 \%$. The multilayer structure at some depth in the constriction was disrupted, as observed by transmission electron microscopy. This was identified as a cause of the low GMR, together with contamination and an oxide layer in the constriction, resulting from ex situ sample rotation. The second generation was fabricated by sputtering of a coupled $\mathrm{Co} / \mathrm{Cu}$ multilayer before etching of the nanohole, giving a proper multilayer at the constriction. Further, the GMR signal from the electrode was shorted by a thick $\mathrm{Cu}$ cap. This did not bring the expected increase of the GMR (ratio $\leqslant 5 \%$ ), indicating that the so-called dead layers and the quality of the interface between the GMR system and the contacting metal were limiting. This interface quality was strongly improved for the third generation of contacts by using in situ rotation, while the question of multilayer quality was avoided by shifting to granular $\mathrm{Co} / \mathrm{Au}$. Granular $\mathrm{Co} / \mathrm{Au}$ in the constriction was obtained by growing a discontinuous Co layer by MBE. The maximum GMR ratio of the granular contacts was $14 \%$, an improvement of a factor 3. These contacts displayed small jumps in the GMR, two-level fluctuations in the resistance time trace and ballistic transport, the latter being evident from phonon peaks in the point-contact spectrum of a high resistance contact. [S0163-1829(99)01537-4]
\end{abstract}

\section{INTRODUCTION}

The discovery of the giant magnetoresistance (GMR) effect of magnetic metallic multilayers ${ }^{1}$ has fuelled numerous studies on giant magnetoresistive systems. The effect consists of a huge drop (up to $\Delta R / R_{\text {sat }}=220 \% ;^{2} \Delta R$ $=$ resistance drop, $R_{\text {sat }}=$ saturated resistance) of the electric resistance on application of a magnetic field. The GMR of multilayers arises from an antiparallel or random orientation of the magnetization of neighboring magnetic layers at zero magnetic field and spin dependent scattering. These effects explain the resistance drop when the magnetization configuration is forced into a parallel one by a magnetic field, causing one "short-circuited" low-resistance spin channel. Most GMR measurements on multilayers have been performed with the current flowing in the plane of the layers, the socalled current in plane (CIP) geometry. While the CIP geometry may be the easiest approach, it is necessary for a more fundamental perspective to have the current flowing perpendicular to the plane of the layers (the so-called CPP geometry). This geometry not only yields a higher GMR ratio $\left(=\Delta R / R_{\text {sat }}\right)$, but the clearer role of the magnetic/ nonmagnetic interfaces allows the relative contributions of interface and bulk spin dependent scattering to be determined. ${ }^{3,4}$ However, the problem of a CPP measurement on the usual short $(\approx 100 \mathrm{~nm})$ and wide $(\approx 1 \mathrm{~mm})$ samples is that the resistance is too small to be measured by conventional techniques. In the first CPP measurements Pratt et al. ${ }^{3}$ used superconducting quantum interference device (SQUID) based electronics to measure the tiny resistance of the multilayer, which was sandwiched between two superconducting Nb contact strips. Such a system only allows measurements at liquid-helium temperatures and relatively low fields $^{3}(H<10 \mathrm{kOe})$. If the lateral dimensions of the multilayer are reduced sufficiently, the need for a SQUID is removed. This can be achieved by using narrower $\mathrm{Nb}$ contact strips, though this still leaves the other limitations mentioned. Alternatively, microfabrication techniques can be utilized to obtain narrow pillars from multilayers. This was pioneered by Gijs, Lenczowski, and Giesbers, ${ }^{5}$ who etched $\mathrm{Fe} / \mathrm{Cr}$ multilayer pillars with cross-sectional areas ranging from $6-130 \mu \mathrm{m}^{2}$. The resulting maximum GMR ratio was $108 \%$, similar to previous CPP values. Meanwhile, other groups used electrodeposition ${ }^{6,7}$ to deposit $\mathrm{Co} / \mathrm{Cu}$ multilayers within nanopores. The maximum CPP GMR ratio of the resulting multilayer nanowires ${ }^{6}$ is $19 \%$, far lower than 170 and $220 \%$, the records for sputtered ${ }^{8}$ and molecular beam epitaxy (MBE) grown ${ }^{2}$ CPP samples, respectively. A large part of 
this difference arises from rough interfaces and/or pin holes, which seem intrinsic to electrodeposited multilayers.

A new way of studying the GMR is by using a point contact or nanoconstriction. Due to the current spreading in a point contact to a multilayer its GMR ratio is expected between the CIP and CPP value and thus should exceed the CIP value. Point contacts of GMR multilayers operating in the ballistic regime (electronic mean free path exceeds the constriction dimensions) are raising interest due to theoretical predictions and their suitability to study intrinsic properties of the electron scattering responsible for the GMR effect. ${ }^{9,10}$ A search for the point-contact GMR was recently made by Tsoi, Jansen, and Bass, ${ }^{11}$ using mechanical point contacts. In this paper we present magnetoresistance measurements on nanofabricated GMR point contacts based on $\mathrm{Co} / \mathrm{Cu}$ and $\mathrm{Co} / \mathrm{Au}$. The contacts were made by introducing these GMR systems into our existing fabrication schemes for metallic point contacts.

This paper is organized as follows. In Sec. II we describe the device fabrication and multilayer growth, postponing individual details and variations to the corresponding sections. In Sec. III we demonstrate a GMR response from point contacts fabricated by deposition of an uncoupled $\mathrm{Co} / \mathrm{Cu}$ multilayer on a silicon nitride membrane with a pre-etched nanohole. The different contributions to the measured resistance are identified in Sec. IV. Of these, the series resistance of the multilayer electrode also gives a GMR signal. Using the solution of Poisson's equation for the specific device structure, we derive the magnitude of this series resistance. Subsequently, in Sec. V we analyze the origin of the GMR measured and its low magnitude $(\leqslant 3 \%)$ on the basis of the relevant resistance contributions and the structural information deduced from transmission electron microscopy (TEM). In Sec. VI MR measurements are presented on contacts fabricated with an alternative procedure, in which a $\mathrm{Co} / \mathrm{Cu}$ multilayer is deposited on the membrane before etching of the hole and the electrode contribution to the GMR is drastically reduced by an effective shunting layer on top of the multilayer. Surprisingly, this procedure, expected to give a better multilayer at the constriction, does not yield a substantial increase of the point-contact GMR (ratio $\leqslant 5 \%$ ). The remainder of Sec. VI inquires into explanations of the low GMR values. Section VII shows results for granular $\mathrm{Co} / \mathrm{Au}$ point contacts. In these we succeeded to achieve pointcontact GMR ratios of up to $14 \%$. It appears that a granular point contact can operate in the ballistic transport regime. In the final section (VIII) we summarize and discuss possible improvements in the fabrication of GMR point contacts.

\section{EXPERIMENTAL}

Our usual way to fabricate ballistic metallic point contacts is to evaporate metal onto both sides of a thin supported $\mathrm{Si}_{3} \mathrm{~N}_{4}$ or $\mathrm{Si}$ membrane with a pre-etched nanohole with a diameter of about $30 \mathrm{~nm}$. The hole in the membrane is made by $e$-beam lithography and reactive ion etching ${ }^{12}$ or wet chemical etching. ${ }^{13}$ Both sides of the membrane are exposed to the evaporation beam, thus filling the hole and forming the device electrodes. For the multilayer contacts we adapted this procedure.

Initially, we used pre-etched holes, in $\mathrm{Si}_{3} \mathrm{~N}_{4}$ membranes,

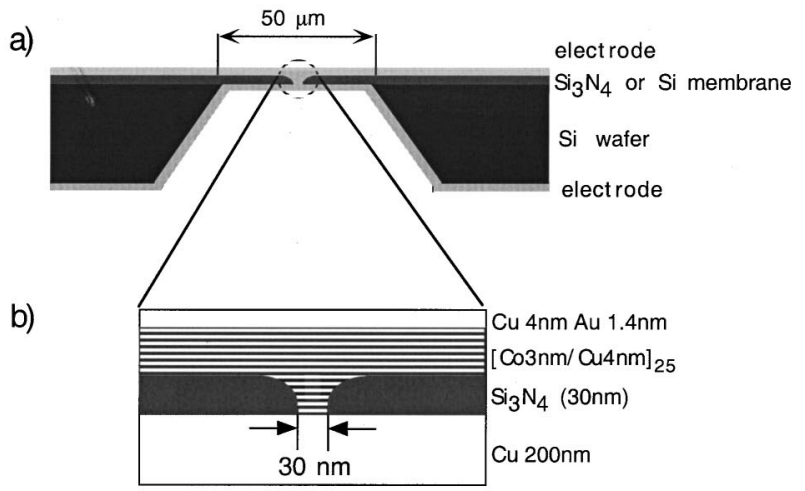

c)

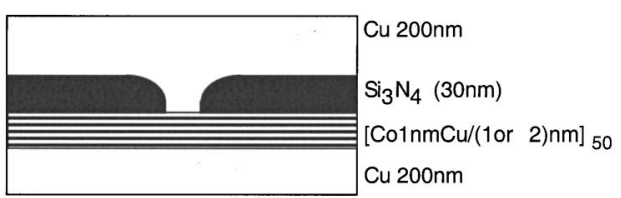

d)

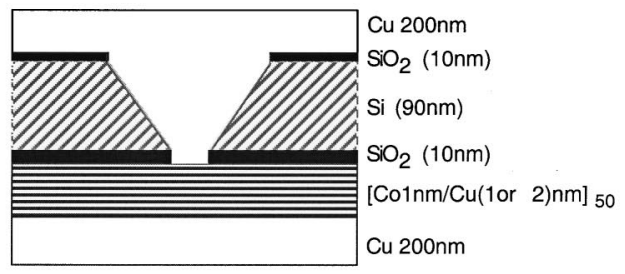

e)

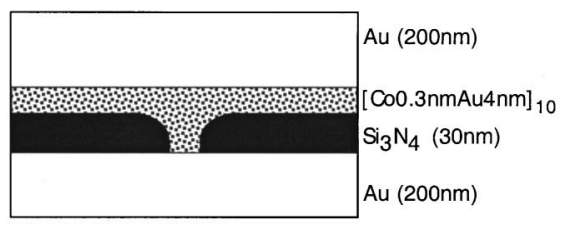

FIG. 1. Schematic cross section of membrane-based point contact (a) and expansions of the central region for contacts based on a pre-etched hole in a $\mathrm{Si}_{3} \mathrm{~N}_{4}$ membrane (b), based on etching after multilayer deposition on a $\mathrm{Si}_{3} \mathrm{~N}_{4}$ membrane (c) and on a $\mathrm{Si}$ membrane (d). (e) shows a granular contact, based on a $\mathrm{Si}_{3} \mathrm{~N}_{4}$ membrane.

while deposition was by MBE using a VG $80 \mathrm{M}$ system. First, a 200-nm-thick $\mathrm{Cu}$ film was deposited on the back of the membrane, to close the hole and form the lower electrode. The sample was then rotated (ex situ) and a multilayer was deposited on the front of the membrane. This gives a layered structure on the membrane and is expected to give such a structure inside the hole, where growth starts on a small $\mathrm{Cu}$ area. A schematic cross section of a contact and an expansion of the central region are shown in Figs. 1(a) and (b). In a second generation of devices we changed the procedure and deposited the multilayer on the back of the membrane before etching the hole. In this case both $\mathrm{Si}_{3} \mathrm{~N}_{4}$ and $\mathrm{Si}$ membranes were used. Another modification is that we shifted from MBE to sputter deposition, which in general yields higher GMR's. In Figs. 1(c) and (d) we show cross sections for these device types. Finally, for the third generation of contacts we returned to $\mathrm{Si}_{3} \mathrm{~N}_{4}$ membranes with a preetched hole, which was used to catch a small number of Co granules from a granular $\mathrm{Co} / \mathrm{Au}$ GMR system [Fig. 1(e)], which was deposited by MBE because of the very accurate control of the layer thickness. 
To enable transport measurements four wires were bonded to the corners of the rectangular multilayer electrode, while two wires were attached to the counter electrode. In this way the device resistance can be measured in a fourpoint geometry and the multilayer resistivity can be determined from a Van der Pauw measurements. Standard fourprobe resistivity measurements on dummy multilayers grown together with the point-contact samples were made for comparison. The contacts were mounted in a ${ }^{4} \mathrm{He}$ flow cryostat equipped with a superconducting magnet. The resistances of the useful contacts were in the range $(0.5-20) \Omega$, corresponding to contact diameters in the range $(100-20) \mathrm{nm}$. The magnetoresistance was measured using ac and dc techniques.

To relate the GMR data to the device structure, for the first generation of contacts cross-sectional TEM was conducted with a Philips CM30T microscope operating at 300 $\mathrm{kV}$. To facilitate preparation of the TEM slices, we used membranes with a series of lines instead of a hole. We assume that the TEM results of the lines are representative of the point contacts. The linewidth was comparable to the hole size of the contacts and the samples were sliced perpendicularly to the lines.

\section{MULTILAYER POINT CONTACTS BASED ON DEPOSITION ON PRE-ETCHED NANOHOLES}

The first contacts were made by MBE growth of an uncoupled $[\mathrm{Co}(3 \mathrm{~nm}) / \mathrm{Cu}(4 \mathrm{~nm})]_{25}$ multilayer on a $\mathrm{Si}_{3} \mathrm{~N}_{4}$ membrane with a pre-etched hole [Fig. 1(b)]. To prevent oxidation the multilayer was capped by a $\mathrm{Cu}(4 \mathrm{~nm}) / \mathrm{Au}(1.4 \mathrm{~nm})$ bilayer. The resistivity of the multilayer is $\rho_{\mathrm{ml}}=8 \mu \Omega \mathrm{cm}$ at $4.2 \mathrm{~K}$. The resistivity of the $200-\mathrm{nm} \mathrm{Cu}$ bottom electrode is $\rho_{\mathrm{Cu}}=0.5 \mu \Omega \mathrm{cm}$. We used uncoupled Co layers, which have a thicker spacer layer than antiferromagnetically coupled layers, but nevertheless have an appreciable GMR of up to $20 \%,{ }^{14}$ as we anticipated that the growth of a multilayer inside and close to the nanohole will be disturbed in comparison to growth on a flat substrate. In particular, inside the hole a rougher multilayer was expected, which for a too thin spacer layer can easily lead to "pinholes" of magnetic material leaking through the spacer. Such pinholes give ferromagnetic coupling between adjacent ferromagnetic layers and thus reduce the GMR.

In Fig. 2 the MR of two multilayer point contacts with resistances of 0.52 and $1.54 \Omega$ is shown. The magnetic field was applied parallel to the layers and thus perpendicular to the constriction axis. Both curves have a clear GMR signal, the GMR ratio $\Delta R / R_{\text {sat }}$ being 10 and $6.2 \%$, respectively. A plot of $\Delta R_{\text {tot }}$ vs $R_{\text {tot }}$ for 17 contacts with clear GMR signals, including those of Fig. 2, is shown in Fig. 3, where the inset gives the relation between $R_{\text {tot }}$ and $2 a$ [see Eq. (2), Sec. IV]. For these contacts $\Delta R / R_{\text {sat }}$ is in the range $2-10 \%$. As a reference we have also measured the CIP GMR of the multilayer electrode of these contacts, using a Van der Pauw geometry. The result is $\Delta R / R_{\text {sat }}=14 \%$ for the transverse geometry (see Fig. 2) and $\Delta R / R_{\text {sat }}=8 \%$ in the longitudinal geometry. The anisotropic magnetoresistance ${ }^{15}$ (AMR) resulting from these values is $5.2 \%$, which is remarkably high. However, it should be noted that the Van der Pauw geometry is not ideal to measure the AMR and affects its magnitude. ${ }^{16}$ The GMR of the point contacts and the CIP GMR display

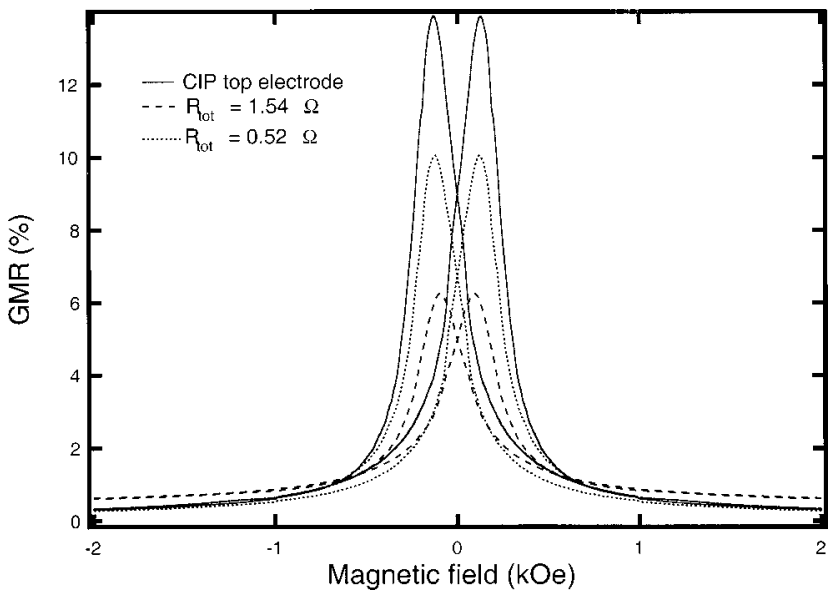

FIG. 2. GMR of two point contacts made by deposition of a $[\mathrm{Co}(3 \mathrm{~nm}) / \mathrm{Cu}(4 \mathrm{~nm})]_{25}$ multilayer on a pre-etched hole in a $\mathrm{Si}_{3} \mathrm{~N}_{4}$ membrane, and CIP GMR of this multilayer, measured at 4.2 $\mathrm{K}$. The field is in the plane of the layers, so that for the point contacts the average current through the constriction is perpendicular to the field.

characteristics expected for a decoupled multilayer: low saturation field $(\approx 1 \mathrm{kOe})$ and coercive nature. The most striking feature of the GMR of the contacts is the low ratio in the range $2-10 \%$ relative to its CIP counterpart (14\%). This is contrary to the expectation for a point contact. To put this result in a proper perspective, we will in the next section consider the various contributions to the measured resistance.

To determine the transport regime of the point contacts we have measured their point-contact spectra $d^{2} I / d V^{2}(V)$, i.e., the bias dependence of the second derivative of the $I-V$ characteristic. For ballistic metallic point contacts $d^{2} I / d V^{2}(V)$ displays peaks at the positions of the maxima in the phonon density of states of the metal. ${ }^{17}$ These peaks arise from emission of phonons by electrons accelerated in the constriction region. For the multilayer point contacts such peaks due to phonon emission were not found. Instead, only

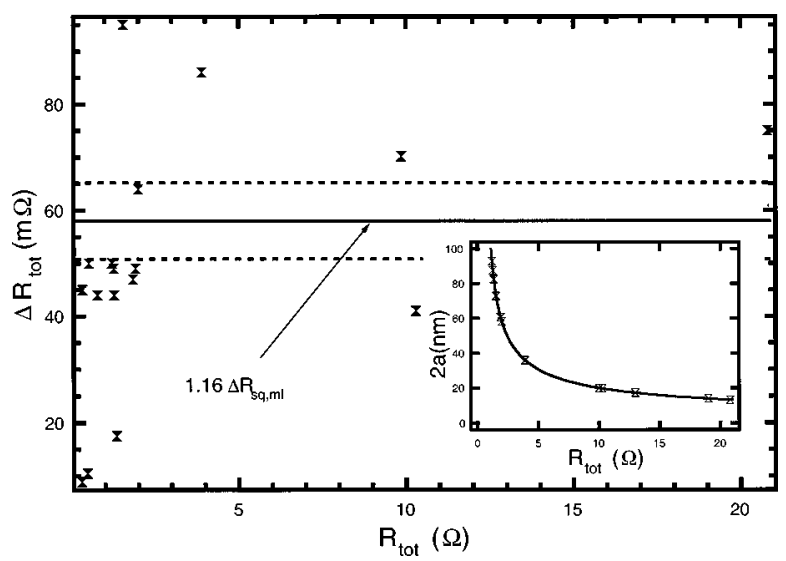

FIG. 3. Resistance change $\Delta R_{\text {tot }}$ due to the GMR effect versus device resistance $R_{\text {tot }}$ for the $[\mathrm{Co}(3 \mathrm{~nm}) / \mathrm{Cu}(4 \mathrm{~nm})]_{25}$ multilayer deposited on a pre-etched hole in a $\mathrm{Si}_{3} \mathrm{~N}_{4}$ membrane, at $4.2 \mathrm{~K}$. The line at $1.16 \Delta R_{\mathrm{sq}, \mathrm{ml}}$ indicates the contribution of the electrode to $\Delta R_{\mathrm{tot}}$. The dashed lines represent the error bar on $1.16 \Delta R_{\mathrm{sq}, \mathrm{ml}}$. The inset gives the relation between the contact diameter and $R_{\text {tot }}$, a number of measured contacts being put on the curve. 


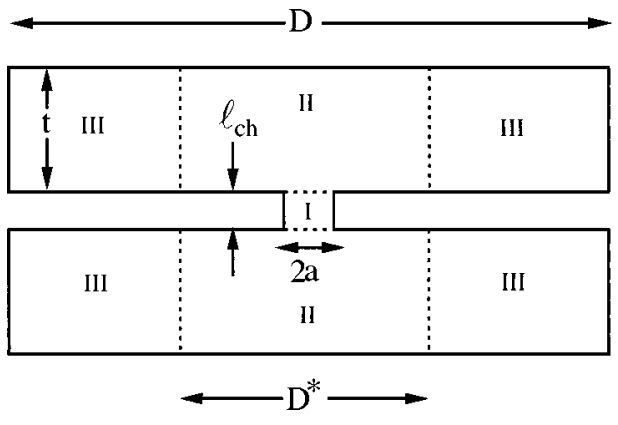

FIG. 4. Model of a point contact with regions I, II, and III, which correspond to the resistance contributions $R_{\mathrm{ch}, \mathrm{ml}}, R_{M, \mathrm{ml}(\mathrm{Cu})}^{*}$ and $R_{\mathrm{el}, \mathrm{ml}(\mathrm{Cu})}$, respectively. Symbols are explained in the text.

a smooth background curve was measured. This indicates that the transport of electrons through the constriction region is diffusive, in agreement with the fact that the elastic mean free path $l_{e}$ in the multilayers is mainly limited by scattering at the interfaces, of which the spacing is much smaller than the constriction diameter $2 a\left(l_{e} \ll 2 a\right)$. This implies ${ }^{17}$ that the ballistic (Sharvin) contribution to the total resistance is much smaller than the diffusive (Maxwell) contribution, so that an MR contribution from the ballistic resistance is expected to be much smaller than the MR from the diffusive resistance. Consequently, the GMR in our point contacts is the usual GMR, wich arises from a mixture of spin dependent interface and bulk scattering, ${ }^{3,4}$ to be contrasted with the ballistic GMR of Schep, Kelly, and Bauer, ${ }^{9}$ which is a band-structure effect.

\section{CONTRIBUTIONS TO THE RESISTANCE OF A MAGNETIC MULTILAYER POINT CONTACT}

Our point contacts differ from an ideal diffusive or Maxwell point contact, ${ }^{18}$ which is a narrow constriction of zero length between two metallic half spaces, operating under the condition $l_{e}<2 a$. Actually, our contacts are a narrow channel between two thin-film electrodes. To fix the situation, let us consider the case of Fig. 1(b). This is repeated in Fig. 4, where three device regions are indicated. The total resistance $R_{\text {tot }}$ is the addition of the resistance of the multilayer channel $R_{\mathrm{ch}, \mathrm{ml}}$ (region I), the three-dimensional (3D) spreading resistances $R_{M, \mathrm{ml}}^{*}$ and $R_{M, \mathrm{Cu}}^{*}$ from the channel to the multilayer electrode and to the copper electrode (regions II) and the 2D spreading resistances $R_{\mathrm{el}, \mathrm{ml}}$ and $R_{\mathrm{el}, \mathrm{Cu}}$ of the electrodes (regions III):

$$
R_{\mathrm{tot}}=R_{\mathrm{ch}, \mathrm{ml}}+R_{M, \mathrm{ml}}^{*}+R_{M, \mathrm{Cu}}^{*}+R_{\mathrm{el}, \mathrm{ml}}+R_{\mathrm{el}, \mathrm{Cu}} .
$$

The asterisk on $R_{M, \mathrm{ml}}^{*}$ and $R_{M, \mathrm{Cu}}^{*}$ indicates that the 3D spreading resistance, due to the thin-film geometry, may deviate from the Maxwell resistance $R_{M}=\rho / 4 a$, which applies to spreading into an infinite half space. The quantity of interest is the point-contact GMR. This comes from the contribution $R_{\mathrm{ch}, \mathrm{ml}}+R_{M, \mathrm{ml}}^{*}$. However, from results on narrow pillars etched from GMR multilayers and contacted with thin film electrodes ${ }^{5,19}$ and from results on mechanical GMR point contacts ${ }^{11}$ it is known that the spurious series resistance of the electrodes can dominate the total resistance. This arises from the potential distribution in the electrodes.
To quantify the potential-distribution effects and the series resistances in our point contacts, we solved Poisson's equation for a structure similar to that of Fig. 4, using finite element methods. ${ }^{20}$ For simplicity we considered only one electrode, which we assumed to be a circular disk of diameter $D$. The model structure thus comprises a 50-nmdiameter cylindrical channel contacting a multilayer disk at its center. To keep the number of elements within the limits of the program, the disk diameter was restricted to $100 \mu \mathrm{m}$. The multilayer was treated as a homogeneous conductor of resistivity $\rho_{\mathrm{ml}}=8 \mu \Omega \mathrm{cm}$. The trivial contribution $R_{\mathrm{ch}, \mathrm{ml}}$ $=\rho_{\mathrm{ml}} l_{\mathrm{ch}} / \pi a^{2}$, which is irrelevant in determining the electrode contribution, was made negligible by setting $\rho_{\text {ch }}$ $=10^{-8} \mu \Omega \mathrm{cm}$, so that the resistance of the structure reduces to $R=R_{M}^{*}+R_{\mathrm{el}}$ (in this section we further drop the index $\mathrm{ml})$.

First, for circumferential current injection (circumference of the disk is equipotential surface), we have determined the inner diameter $D^{*}$ of region III. In this region of cylindrical current spreading (implying for the electric field $\mathbf{E}=-\boldsymbol{\nabla} V$ $\propto 1 / r_{2 D}$, for $\left.r_{2 D} \geqslant D^{*}\right)$ the in-plane radial potential profiles $V\left(r_{2 D}\right)$ for different levels in the disk coincide and obey $V\left(r_{2 D}\right)-V\left(D^{*}\right) \propto \ln \left(r_{2 D} / D^{*}\right)$. For $r_{2 D} \leqslant D^{*}$ the in-plane profiles correspond to 3D spreading and thus do not coincide. So $D^{*}$, for $a \ll D^{*}$ expected to be proportional to the electrode thickness $t$, should be the merging point of calculated profiles for different levels. For disks with $D=50 \mu \mathrm{m}$ and $t=100,200$, and $300 \mathrm{~nm}$ the merging point was determined as $D^{*}=3 t$, leading to $R_{\mathrm{el}}=\left(R_{\mathrm{sq}} / 2 \pi\right) \ln (D / 3 t) . R_{\mathrm{sq}}$ is the sheet resistance of the multilayer.

Further, also for circumferential current injection and using $R=R_{M}^{*}+\left(R_{\mathrm{sq}} / 2 \pi\right) \ln (D / 3 t)$, we extracted the value of $R_{M}^{*}$ from the resistance for disks with $D=10,50$, and 100 $\mu \mathrm{m}$ and $t=200 \mathrm{~nm}$. The result is $R_{M}^{*}=0.70 \Omega$. This is smaller than $R_{M}=\rho / 4 a=0.80 \Omega$. The difference arises from the finite thickness of the electrode. A better approximation for this case is $R_{M}^{*} \approx(\rho / 4) \int_{a}^{t} d r_{3 D} /\left(r_{3 D}\right)^{2}=\rho(1 / 4 a-1 / 4 t)$ $\approx 0.70 \Omega$, in agreement with the value from the finite element calculation.

Finally, we calculated the case of point injection of the current into the disk by attaching a current lead to its border at point $P$. Four-point measurements on a point contact then correspond to "sensing" the voltage developed at point $Q$ at the border. It is found that the voltage between point $Q$ and the channel depends on the relative orientation $\varphi$ of the positions $P$ and $Q$ of the leads at the border. Here $\varphi$ $=\angle(P O Q), O$ being the center of the disk. In Fig. 5(a) we show calculated equipotential lines in a small region of a $50-\mu \mathrm{m}$-diameter disk, for the plane through the constriction axis and point $P$, which is at the left (outside the region drawn). We find that the potential distribution in region II is unaltered with respect to that of circumferential current injection. In region III, however, it is clearly modified as a result of the asymmetric injection. This is seen in Fig. 5(b), which shows the equipotential lines on a larger scale, in the plane of the multilayer-membrane interface. Again, current is injected at the left. The lines indicate that the radial profile $V\left(r_{2 D}\right)$ is steepest for $\varphi=0^{\circ}$. When $\varphi$ increases, $V\left(r_{2 D}\right)$ becomes less steep. For $\varphi=180^{\circ}$, the situation of the experiment, the slope is minimum, but not zero. This corresponds 


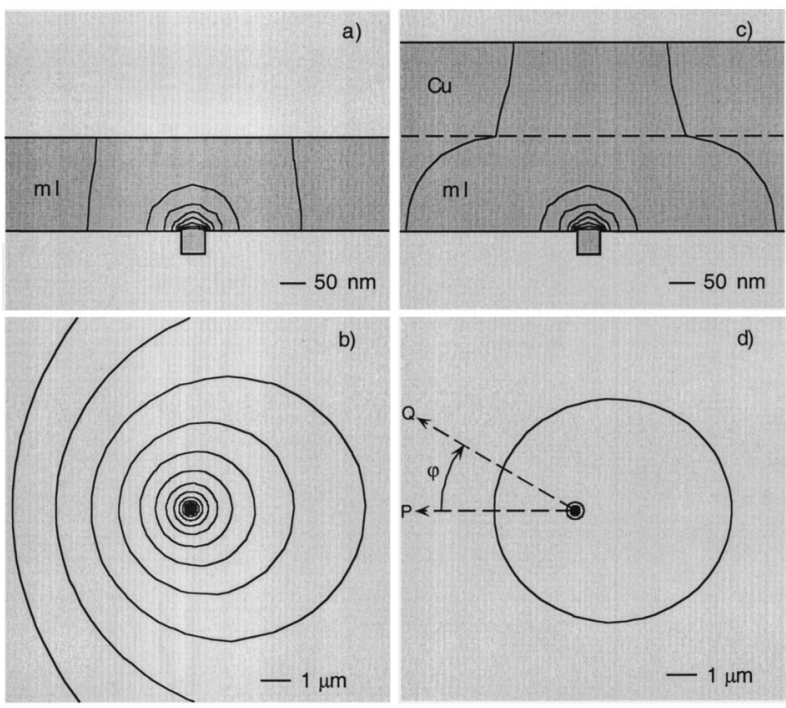

FIG. 5. Potential distributions for a 50- $\mu$ m diameter, 200-nmthick electrode and for point injection of the current $(2 \mathrm{~mA}$, injected at the border, on the left), obtained with finite element calculations. (a) and (b) are for a multilayer ( $\mathrm{ml}$ ) without shunting layer, showing equipotential lines in a plane through the constriction axis (equipotential spacing $0.17 \mathrm{mV}$ ) and in the plane at the multilayer/ membrane interface (equipotential spacing $0.05 \mathrm{mV}$ ), respectively. (c) and (d) are for a multilayer with a 200-nm-thick shunting layer, for the same planes and the same equipotential spacings as in (a) and (b). The potential of the channel, visible in (a) and (b), is $0 \mathrm{mV}$. $\varphi=\angle(P O Q)$ is defined in (d). The device sections in (a) and (c) approximately correspond with region III.

to a measured electrode contribution to the device resistance. These results lead to the generalized expression $R=R_{M}^{*}$ $+K\left(D, D^{*}, \varphi\right) R_{\mathrm{sq}}$. Here $K\left(D, D^{*}, \varphi\right)$ is a geometrical factor, which takes into account the size and thickness of the electrode and the relative orientation of the leads at the border. Using the value of $R_{M}^{*}$ extracted from the circumferential case, we have determined $K\left(D, D^{*}, \varphi\right)$ for disks of different diameter and with $t=200 \mathrm{~nm}$, and for several values of $\varphi$. When plotted versus $\ln \left(D / D^{*}\right)$ the $K$ values show linear behavior. To find $K\left(D, D^{*}, \varphi\right)$ for $D=3 \mathrm{~mm}$, which approximately corresponds to the multilayer electrode of the contacts of Sec. III, we have extrapolated the results to this diameter. For $\varphi=180^{\circ}$ we find $K\left(3 \mathrm{~mm}, 0.6 \mu \mathrm{m}, 180^{\circ}\right)$ $=1.16(t=200 \mathrm{~nm})$, giving $R_{\mathrm{el}}=0.46 \Omega$. For comparison, we note that the expressions $R_{\mathrm{el}}=R_{\mathrm{sq}} \ln (D / 4 t) / 2 \pi$ and $R_{\mathrm{el}}$ $=R_{\mathrm{sq}}[\ln (D / 4 t)-\ln (1-\cos \varphi)] / 2 \pi$, advocated in Refs. 11 and 21 , yield $K=1.31$ and $K=1.20$, respectively. ${ }^{22}$

The series resistance $R_{\mathrm{el}}=K \quad R_{\mathrm{sq}}$ suggests the use of a highly conducting layer on top of the multilayer, to shunt this contribution and thus the CIP GMR of the multilayer. For this purpose one can use a thick Cu layer with $\rho_{\mathrm{Cu}} \ll \rho_{\mathrm{ml}}$ or a superconducting layer. In Figs. 5(c) and 5(d) we show the resulting equipotential lines in case of a 200-nm-thick $\mathrm{Cu}$ shunting layer with $\rho=0.5 \mu \Omega \mathrm{cm}$ on top of the multilayer. In the multilayer the very inner region is unaffected [Fig. 5(c)], but further away the potential landscape is clearly less steep. The equipotential lines in the $\mathrm{Cu}$ layer indicate that the current is strongly drawn to this layer. Figure 5(d) indicates on a larger scale that the potential drop in the electrode is strongly reduced. The total resistance for the shunted case is $\approx 0.72 \Omega$, which is only weakly dependent on $\varphi$. The slight increase above $R_{M}^{*}=0.70 \Omega$ is due to the series resistance of the $\mathrm{Cu}$.

\section{ORIGIN OF THE GMR OF CONTACTS BASED ON DEPOSITION ON PRE-ETCHED NANOHOLES}

Due to the weak logarithmic dependence of $K\left(D, D^{*}, \varphi\right)$ on $D^{*}=3 t$, we may adopt $K=1.16$, the value for $t$ $=200 \mathrm{~nm}$, for the contacts of Sec. III $(t=175 \mathrm{~nm})$. For these contacts the ratio $\rho_{\mathrm{ml}} / \rho_{\mathrm{Cu}}$ is 16 , so that in Eq. (1) $R_{M, \mathrm{Cu}}^{*}$ and $R_{\mathrm{el}, \mathrm{Cu}}^{*}$ can be neglected. For a cylindrical channel this leads to

$$
R_{\mathrm{tot}}=\frac{\rho_{\mathrm{ml}}}{4 a}\left[\frac{4}{\pi} \frac{l_{\mathrm{ch}}}{a}+\left(1-\frac{a}{t}\right)+1.16 \frac{4 a}{t}\right] .
$$

For typical constriction diameters $2 a \approx 30 \mathrm{~nm}$ the last term in Eq. (2) is appreciable compared to the other terms, so that it contributes a spurious CIP GMR signal. For the multilayer electrode $\rho_{\mathrm{ml}}=(8 \pm 1) \mu \Omega \mathrm{cm}$ (value at $20 \mathrm{kOe}$ ), giving $R_{\mathrm{el}, \mathrm{ml}}=(0.53 \pm 0.07) \Omega$ for the last term. Due to the radial current spreading in the plane of the electrode, we have to take the average of the longitudinal and transverse GMR's of the multilayer as measured in the Van der Pauw geometry (see Sec. III), giving $11 \%$. This then implies $\Delta R_{\mathrm{el}, \mathrm{ml}}$ $=58 \mathrm{~m} \Omega$. In Fig. 3 it is seen that quite a number of points bunch close to $53 \mathrm{~m} \Omega$, so that for these points probably $\Delta R_{\mathrm{el}, \mathrm{ml}}$ accounts for $\Delta R_{\mathrm{tot}}$. Interestingly, however, for four devices $\Delta R_{\text {tot }}$ clearly exceeds $\Delta R_{\text {el,ml }}$. In these cases a substantial part of the GMR originates from the constriction region. For example, for the 1.54- $\Omega$ contact $\Delta R_{\text {tot }}=95 \mathrm{~m} \Omega$. After correction for the electrode contributions to $R_{\text {tot }}$ and $\Delta R_{\text {tot }}$, the corresponding point-contact GMR ratio is $3 \%$, the highest value for this generation of contacts.

Another indication of the relative importance of the terms in Eq. (2) can be obtained from the dependence on the field orientation of the saturated magnetoresistance of a device, which is dominated by the AMR. ${ }^{15}$ According to the AMR effect $\rho_{\text {sat }, \|}$ exceeds $\rho_{\text {sat }, \perp}$, while in a measurement the current through $R_{\mathrm{el}, \mathrm{ml}}$ flows perpendicularly to the current through $R_{\mathrm{ch}}$ and $R_{M, \mathrm{ml}}$. This property enables determination of the device section dominating the AMR. Here $\rho_{\text {sat }, \|}, \rho_{\text {sat }, \perp}$ are saturated resistivities for $I \| M_{\text {sat }}, I \perp M_{\text {sat }}$, respectively ( $M_{\text {sat }}$ is the saturated magnetization; $M_{\text {sat }} \| H$ ). MR curves for the $0.52-\Omega$ point contact of Fig. 2 are shown in Fig. 6, for $\theta=0^{\circ}, 80^{\circ}, 90^{\circ}$, where $\theta$ is the angle between the applied field and the constriction axis. For this contact $\Delta R_{\text {tot }}$ is close to $\Delta R_{\mathrm{el}, \mathrm{ml}}$. The maximum of the saturated resistance occurs for $\theta=0^{\circ}$ and the minimum for $\theta=90^{\circ}$. This agrees with an AMR of the constriction region, where the current flows parallel to the constriction axis (taking for $R_{M \text {, ml }}$ the average current direction), and not with an AMR of the multilayer electrode. Thus the AMR of $R_{\text {tot }}$ is dominated by the constriction region, while the GMR, as argued above, is determined by the multilayer electrode. In view of the disrupted structure in the channel this can very well be the case. In Fig. 6 a strong broadening is present in the curve for $\theta=0^{\circ}$. This demonstrates that the direction perpendicular to the layers is a hard direction for the magnetization. For $\theta=0^{\circ}$ there is also a shoulder in the curve. From our previous work ${ }^{23}$ we know that this shoulder is due to a nonuniformity of the 


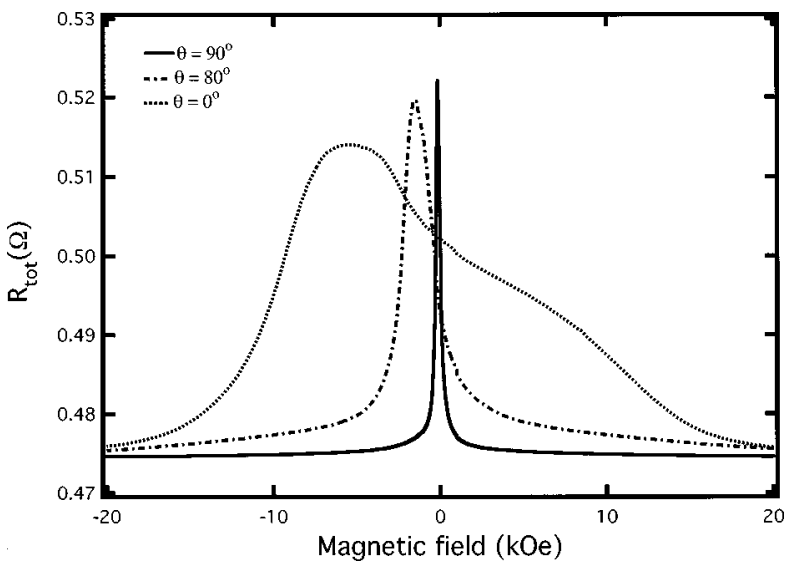

FIG. 6. GMR curves for the $0.52-\Omega$ contact, for different angles $\theta$ between the field and the constriction axis. Note that, due to the AMR effect of the channel, a reduction in saturated resistance is recorded as $\theta$ increases from $0^{\circ}-90^{\circ}$. The sweep direction is from positive to negative fields.

anisotropy and the easy axis within the magnetic layers. The slight differences in the orientation of the layers evident from the TEM results discussed below agree with this.

The dominance of the constriction for the AMR effect may indicate that Eq. (2) overestimates the electrode contribution for the $0.52-\Omega$ contact, and thus underestimates $R_{\mathrm{ch}}$ $+R_{M, \mathrm{ml}}$ and its contribution to the GMR. This may arise from flowed out silver paint, that we use for wire bonding. Flowed out silver paint, which for our way of handling has a typical sheet resistance of $10 \mathrm{~m} \Omega$, has the same effect as the copper shunting layer discussed in the previous section. Shunting of $R_{\mathrm{el}, \mathrm{ml}}$ by a silver-paint film may also play a role for other contacts and explains that for some devices $\Delta R_{\text {tot }}$ $<\Delta R_{\mathrm{el}, \mathrm{ml}}$. However, though the point-contact GMR may be larger than implied by Eq. (2), even if the whole measured GMR is attributed to the point contact, it is still falls short of the value expected.

Having shown that our devices yield a point-contact GMR, the question arises why this GMR is smaller than the CIP GMR. To answer this, we consider the TEM micrographs in Figs. 7(a) and 7(b), which gives structural information on the material in the constriction region. These bright-field images were taken slightly out of focus in order to increase the visibility of the different layers. Figure 7(a) shows that away from the constriction a regular multilayer structure is present on the membrane. The waviness of the multilayer, which arises as a result of a columnar growth mode, is similar to that found by others. ${ }^{24}$ The dimensions of the columns are mostly larger than the individual layer thickness, as judged from the dark regions, which correspond to strongly diffracting single crystals. Towards the constriction the layered structure follows its tapered shape. Even inside the channel the layers sequence can be recognized [Fig. 7(b)]. Deeper in the constriction disruption of the multilayer is seen. On close inspection one can see segregated Co and $\mathrm{Cu}$ regions. These, however, do not occur in a regular sequence. Lattice imaging of the grain structure of the specimen showed that the grains in the constriction are much smaller $(4-10 \mathrm{~nm})$ than those of the undisturbed multilayer $(20-50 \mathrm{~nm})$.

The TEM results give us some clues to understand the (a)

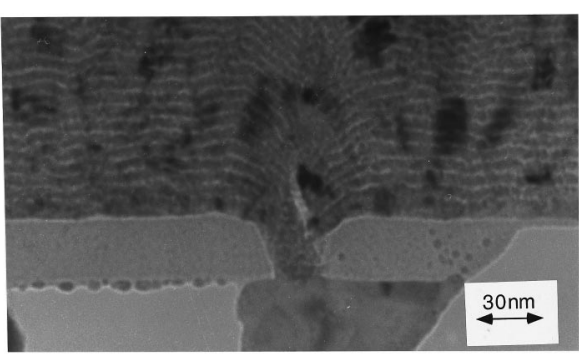

(b)

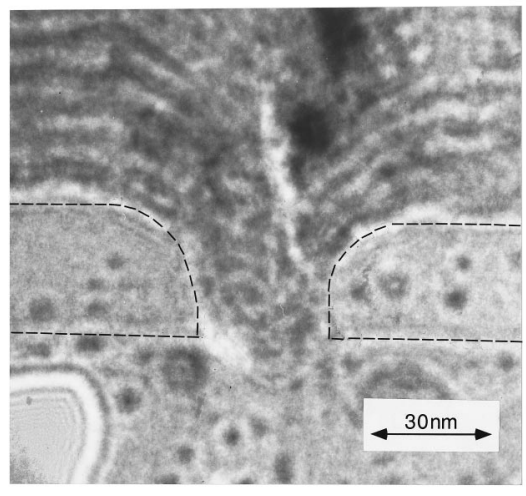

FIG. 7. (a) Bright-field cross-sectional TEM micrograph (slightly out of focus) of the $[\mathrm{Co}(3 \mathrm{~nm}) / \mathrm{Cu}(4 \mathrm{~nm})]_{25}$ multilayer, deposited on a $\mathrm{Si}_{3} \mathrm{~N}_{4}$ membrane with an etched line. In the line the $\mathrm{Co} / \mathrm{Cu}$ comes into contact with the 200-nm $\mathrm{Cu}$ layer deposited on the back of the membrane. The expanded view in (b) shows that there is some continuation of the multilayer sequence inside the line, but that the multilayer itself is disrupted.

electrical data. The segregated $\mathrm{Co}$ and $\mathrm{Cu}$ in the channel will contribute a GMR signal of mixed CPP and CIP character which is smaller than the GMR coming from the regular multilayer. In addition, the disorder will cause the channel to have a higher saturated resistivity than the regular multilayer. Also, between Co regions in the disrupted material ferromagnetic coupling may exist. The conformal nature of the layers at the rounded edges of the constriction leads to a CIP-like contribution, as the current through the diffusive contacts follows a similar path around the constriction edges. Taking all these factors together, one may expect a GMR ratio below the CIP value. Our finding of a reduced point-contact GMR is similar to that of Tsoi, Jansen, and Bass ${ }^{11}$ for mechanical $\mathrm{Co} / \mathrm{Cu}$ point contacts. Also these authors attribute the effect to disorder in the $\mathrm{Co} / \mathrm{Cu}$ system, which in their case is the result of damage made by the tip. We note that the higher (but unknown) resistivity of the channel, when accounted for in Eq. (2), will lead to larger constriction diameters.

Another possible cause of the small GMR is a high resistance of the interface between the $\mathrm{Cu}$ electrode and the $\mathrm{Co} / \mathrm{Cu}$ system deposited on top of that. Such a resistance may result from contamination and oxidation of the $\mathrm{Cu}$ surface during the ex situ rotation of the sample after deposition of the $\mathrm{Cu}$ electrode. The rotation procedure gives an exposure to air of the sample during $20 \mathrm{~min}$. In view of the rapid initial oxidation of $\mathrm{Cu}^{25}$ this results in an estimated oxide thickness of $0.5 \mathrm{~nm}$. At room temperature the oxide formed is $\mathrm{Cu}_{2} \mathrm{O},{ }^{25}$ which is a semiconductor. At low temperature the interfacial oxide layer will have a negative effect on the device performance, the extent of which is difficult to quantify. 


\section{MULTILAYER POINT CONTACTS BASED ON DEPOSITION BEFORE ETCHING OF THE NANOHOLE}

In the second generation of contacts we removed the most obvious shortcoming of the contacts, i.e., the disrupted structure of the multilayer at the constriction. The modified process is characterized by deposition of the $\mathrm{Co} / \mathrm{Cu}$ multilayer on the back of the membrane before the nanohole is etched from the front. From the regularity of the multilayer on the flat region of the membrane in Fig. 7 we thus expected at the constriction a much better quality of the multilayer. A condition is that the etching of the hole does not damage the multilayer. Further, the multilayer of the new contacts was covered with a 200-nm-thick $\mathrm{Cu}$ layer with $\rho_{\mathrm{Cu}}$ $=0.5 \mu \Omega \mathrm{cm}$. As demonstrated in Sec. IV, such a thick overlayer effectively shorts the electrode contribution to the resistance, so that in Eq. (2) only the first two terms are left. Finally, the depositions were done with sputtering, as this yields higher GMR ratios than MBE. After etching of the hole towards the multilayer, the devices are completed by deposition of $\mathrm{Cu}$ on the reverse side, to fill the hole and form the 200-nm-thick counter electrode.

The contacts are based on both $\mathrm{Si}_{3} \mathrm{~N}_{4}$ and $\mathrm{Si}$ membranes (see Sec. II). Holes in $\mathrm{Si}_{3} \mathrm{~N}_{4}$ membranes [Fig. 1(c)] are etched in an $\mathrm{SF}_{6}$ plasma. ${ }^{12}$ For $\mathrm{Si}$ membranes the critical etch of the holes is that of the final thin oxide [Fig. 1(d)], which is done in an aqueous solution of HF. ${ }^{13}$ The structure of the multilayer electrode is $\left[\mathrm{Co}(1 \mathrm{~nm}) / \mathrm{Cu}\left(t_{\mathrm{Cu}}\right)\right]_{50} / \mathrm{Cu}$ $(200 \mathrm{~nm})$, with $t_{\mathrm{Cu}}$ set to 1 or $2 \mathrm{~nm}$, corresponding to the first and second peak in the anti-ferromagnetic (AF) coupling, respectively. The multilayers resistivities (at $4.2 \mathrm{~K}$ ) are $\rho_{\mathrm{ml}}$ $=15 \mu \Omega \mathrm{cm}$ and $\rho_{\mathrm{ml}}=10 \mu \Omega \mathrm{cm}$, for $t_{\mathrm{Cu}}=1 \mathrm{~nm}$ and $t_{\mathrm{Cu}}$ $=2 \mathrm{~nm}$, respectively. Deposition of a multilayer started with the $\mathrm{Cu}$ layer. For either thickness of the coupling layer two sets of samples were grown: one without buffer and one with a 5-nm $\mathrm{Cu}$ buffer. The purpose of the buffer is reduction of possible etch damage to the multilayer. For high resistance contacts, which have a small probed volume, a multilayer on a 5-nm buffer may be outside the spatial range where most of the voltage drops, giving a less sensitive measurement of the GMR. In view of the $\mathrm{Cu}$ etch rate in the $\mathrm{SF}_{6}$ plasma, and taking into account the overetch time in the etching of the hole, at most $0.7 \mathrm{~nm} \mathrm{Cu}$ is consumed in the $\mathrm{SF}_{6}$ plasma. The $\mathrm{Cu}$ etch rate in the $\mathrm{HF}$ etchant and the exposure to this etchant are such that not more than a few monolayers of $\mathrm{Cu}$ are etched. In either case we expected the load on the multilayer weak enough to not really be harmful to its quality.

For each of the four types, of this second generation we have measured the MR of several point contacts. Globally, the results for contacts based on nitride and silicon membranes were rather similar. Examples of point-contact GMR's are given in Fig. 8, for either membrane type. The magnetic field is oriented in the plane of the layers. For each type of layered structure device-to-device variations were seen in the detailed shape of the GMR curve, as expected for local probing of the magnetization configuration. Devicespecific details are seen in the GMR curve of the $2.5 \Omega$ contact in Fig. 8, which shows small resistance jumps. These jumps are reflections of sudden changes of the magnetization configuration of the multilayer in the constriction region.

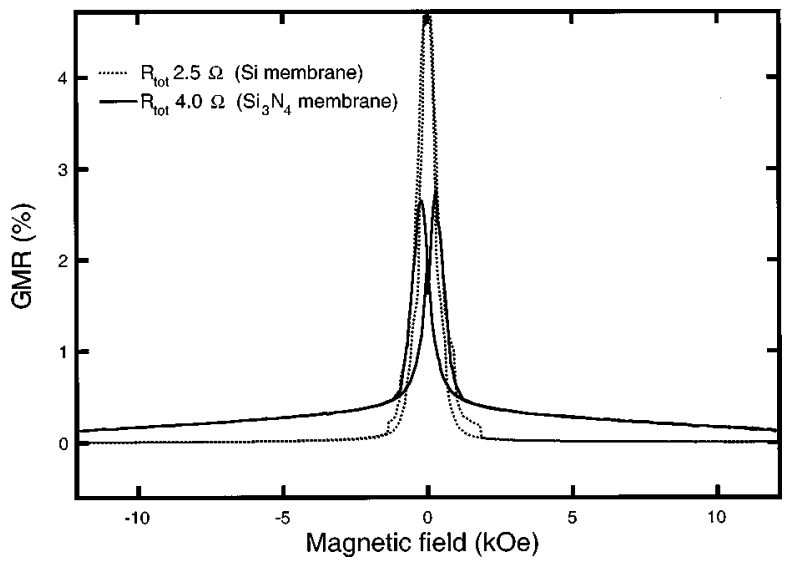

FIG. 8. GMR of contacts based on deposition of [Co $(1 \mathrm{~nm}) / \mathrm{Cu}(2 \mathrm{~nm})]_{50}$ multilayer on $\mathrm{Si}_{3} \mathrm{~N}_{4}$ and $\mathrm{Si}$ membrane, demonstrating that the deposition-before-etching procedure works. Due to the shunting layer the measured GMR is directly the pointcontact GMR. The curve of the 2.7- $\Omega$ contacts has jumps. $T$ $=4.2 \mathrm{~K}$.

The data of all measured contacts indicate that the yield of contacts with a proper GMR signal is higher for contacts with $t_{\mathrm{Cu}}=2 \mathrm{~nm}$. In search of further systematics in the data we plotted $\Delta R_{\text {tot }}$ versus $R_{\text {tot }}$, for the different device types. For this generation of contacts $\Delta R_{\text {tot }}$ is the GMR signal coming from $R_{M, \mathrm{ml}}^{*}$, while $R_{\mathrm{tot}} \approx R_{M, \mathrm{ml}}^{*}$, due to the property $\rho_{\mathrm{Cu}} / \rho_{\mathrm{ml}} \ll 1$. This implies the relation $\Delta R_{\mathrm{tot}} \approx \mathrm{GMR}_{\mathrm{pr}} R_{\text {tot }}$ $\left(\mathrm{GMR}_{\mathrm{pc}}\right.$ is the point-contact GMR ratio, supposed constant among devices of one type). The resulting plots, due to spread in the data points, do not show a proper linear behavior, but an average GMR ratio of up to $5 \%$ can be extracted from them. This is still much lower than the CIP GMR ratios of the control samples produced in the same run, which are 55 and $30 \%$ at $4.2 \mathrm{~K}$ for the first and second peak, respectively.

The modifications of the process, in particular deposition before etching, apparently have not led to an increase of the point-contact GMR. In this several factors can play a role. The first is damage of the multilayer, resulting from etching of the hole. For nitride based contacts damage points to ion bombardment during the $\mathrm{SF}_{6}$ etch. However, the bias voltage of the etch is only $10 \mathrm{~V}$, making ion-bombardment damage highly unlikely. As for contacts based on Si membranes, we note that $\mathrm{HF}$ virtually does not attack $\mathrm{Cu}$ and that it has no access to deeper layers. In connection to this it is remarkable that contacts based on nitride and silicon membranes yield similar GMR ratios, in spite of the different etch. This suggests that etch damage is not a major effect. Another factor is oxidation of the $\mathrm{Cu}$ surface after etching of the hole. Since etching is done ex situ, formation of a $\mathrm{Cu}_{2} \mathrm{O}$ layer on the initial $\mathrm{Cu}$ of the multilayer still is a disadvantage of this process. Finally, the so-called dead layers may reduce the point-contact GMR. These first few bilayers of the multilayer are known to be worse than the subsequent layers and can even be "dead" in relation to the GMR, i.e., they are ferromagnetically coupled. This effect is negligible in a CIP or regular CPP measurement on a multilayer with many bilayers, but will be pronounced for a point contact, which probes the direct vicinity of the constriction most sensitively. That 
contacts operating in the second peak of the AF coupling yield better GMR signals than those operating in the first peak may be related to the dead-layer effect, which is likely to be weaker for second peak samples.

\section{GRANULAR Co/Au POINT CONTACTS}

The results in the previous sections suggest a fabrication procedure which avoids dead layers and an oxide at the constriction. This implies deposition of the insulating layer of the contact on top of the multilayer without breaking the vacuum and in situ etching of the nanohole in this layer, followed by contacting of the multilayer through the hole. This is a very demanding procedure, which brings several other complications. To avoid these we have chosen a different route, and made granular GMR point contacts. Granular point contacts have a constriction region consisting of singledomain ferromagnetic granules embedded in a nonmagnetic metal. They resemble the contacts with the disrupted multilayer (see Fig. 7). The difference is that the Co regions in granular contacts can be made in a controlled way. Further, the granules can be small enough to accommodate several of them in the constriction, suggesting the use of preetched holes. By applying in situ sample rotation, for which a facility became available in this stage, an interfacial oxide is avoided.

It is well known ${ }^{26}$ that a granular GMR system with single-domain granules exhibits superparamagnetism, characterized by a blocking temperature $T_{b}$. Above $T_{b}$ the system is seemingly paramagnetic if the time scale of magnetization reversal of the granules is shorter than the temporal resolution of the apparatus probing the magnetic state, while below $T_{b}$ the system behaves more like a ferromagnetic system, exhibiting hysteresis in an external magnetic field. By defining a point contact to a granular system a small number of magnetic granules or clusters can be probed in a resistance measurement. For the third generation of devices we followed this approach to study granular $\mathrm{Co} / \mathrm{Au}$ point contacts. The solubility of $\mathrm{Co}$ in $\mathrm{Au}$ is poor, so that the condition for getting Co granules in a pure Au matrix can be fulfilled.

The granular contacts were made using MBE deposition and membranes with pre-etched holes. We returned to MBE because of its accurate control of the layer thickness and thus of the magnetic particle size. ${ }^{27}$ We deposit a 200 -nm-thick Au film on the back of the membrane, to close the hole and form the lower electrode. The sample is then rotated in situ, followed by deposition of a $[\mathrm{Co}(0.3 \mathrm{~nm}) / \mathrm{Au}(4 \mathrm{~nm})]_{10}$ multilayer on the front. The upper electrode is completed with a 200-nm-thick Au shunting layer. This gives a sheet resistance of the capped multilayer of $50 \mathrm{~m} \Omega$, so that the measured device resistance effectively is that of the channel. Two types of contacts were fabricated: types A and B. The $\mathrm{Au}$ growth rate was $0.07 \mathrm{~nm} / \mathrm{s}$ for each type, while the Co growth rate was $0.01 \mathrm{~nm} / \mathrm{s}$ for type A and $0.03 \mathrm{~nm} / \mathrm{s}$ for type $\mathrm{B}$, all at $300 \mathrm{~K}$. Co layers as thin as $0.3 \mathrm{~nm}$ (nominally) are discontinuous, resulting in a granular structure. For a nominal Co thickness of $0.3 \mathrm{~nm}$ deposited on a flat substrate, deposition rates of $0.01 \mathrm{~nm} / \mathrm{s}$ and $0.03 \mathrm{~nm} / \mathrm{s}$ yield clearly different GMR's and Co cluster sizes of 5 and $4 \mathrm{~nm},{ }^{27}$ respectively. This gives up to several tens of clusters in their constriction. A schematic cross section of a granular contact is shown in Fig. 1(e).
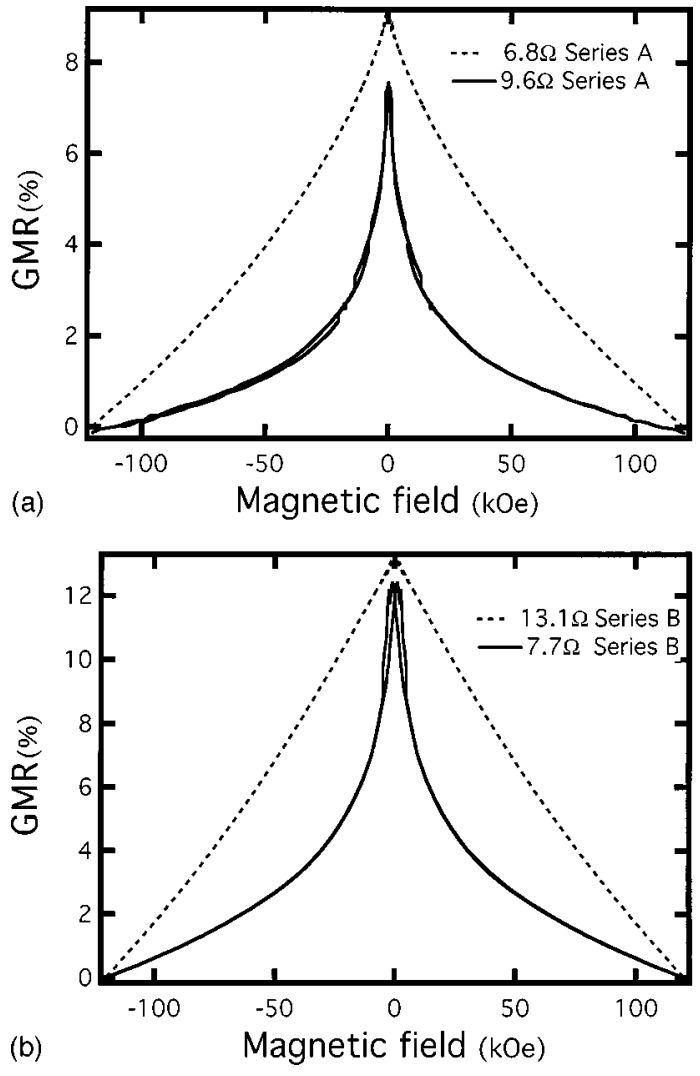

FIG. 9. GMR curves at $4.2 \mathrm{~K}$ of $\mathrm{Co} / \mathrm{Au}$ granular point contacts resulting from deposition of $[\mathrm{Co}(0.3 \mathrm{~nm}) / \mathrm{Au}(4 \mathrm{~nm})]_{10}$ multilayer. Resistance values and device types are shown inset.

In Figs. 9(a) and 9(b) we show two GMR curves for either contact type, measured at $4.2 \mathrm{~K}$. The GMR ratio ${ }^{28}$ of $14 \%$ of the 6.6- $\Omega$ type-B contact (Table I), the maximum for these

TABLE I. Contact type, resistance, GMR ratio, the magnetic nature $(\mathrm{SP}=$ superparamagnetic, $\mathrm{F}+\mathrm{SP}=$ mixed ferromagnetic and superparamagnetic) and the number of clusters in the channel for the $\mathrm{Co} / \mathrm{Au}$ contacts deposited with Co rates of $0.001 \mathrm{~nm} / \mathrm{s}$ (type A) and $0.03 \mathrm{~nm} / \mathrm{s}$ (type B). Note that the number of clusters was estimated using the resistivity and cluster size deduced in Ref. 27, assuming a Co volume fraction based on the layer thicknesses. The error in the number of clusters relates to the error in the resistivity.

\begin{tabular}{ccccc}
\hline \hline $\begin{array}{c}\text { Contact } \\
\text { type }\end{array}$ & $\mathrm{R}(\Omega)$ & GMR $\%$ & $\begin{array}{c}\text { Magnetic } \\
\text { status }\end{array}$ & $\begin{array}{c}\text { No. of } \\
\text { Co clusters } \\
( \pm 15 \%)\end{array}$ \\
\hline A & 5.5 & 7.0 & F+SP & 22 \\
A & 6.8 & 9.0 & SP & 18 \\
A & 8.8 & 6.0 & SP & 14 \\
A & 9.6 & 7.5 & F+SP & 14 \\
B & 5.5 & 8.0 & F+SP & 34 \\
B & 6.0 & 14 & F+SP & 31 \\
B & 6.6 & 14 & F+SP & 29 \\
B & 7.7 & 12 & F+SP & 25 \\
B & 8.0 & 13 & F+SP & 25 \\
B & 13.1 & 13 & SP & 16 \\
B & 16.2 & 11 & SP & 13 \\
B & 22.0 & 5.5 & F+SP & 10 \\
\hline \hline
\end{tabular}


granular contacts, means a substantial improvement over the $5 \%$ GMR ratio of the $\mathrm{Co} / \mathrm{Cu}$ point contacts of Sec. VI. For either type, in general, the GMR curve can be very different in shape. In a number of cases the GMR curve is rather steep close to $H=0$, shows hysteresis and a levelling off at higher fields (in Fig. 9: 9.6- $\Omega$ type A, 7.7- $\Omega$ type B). In other cases the slope variation of the GMR curve is small or even negligible and no hysteresis is observed (in Fig. 9: 6.8- $\Omega$ type A, 13.1- $\Omega$ type B). A small or negligible slope variation arises when solely superparamagnetic Co clusters are present in the constriction, while a rather strong slope variation indicates that a mixture of both superparamagnetic and ferromagnetic clusters is present. In Table I we have compiled the characteristics of the contacts measured, including an estimation of the number of Co clusters within the constriction. This estimation assumes that the cluster size remains unchanged compared to that determined from depositions on flat substrates. Both for type A and type B a correlation between the contact resistance and the GMR ratio, the GMR shape or the resistance change between zero field and high field cannot be deduced from the data.

It is surprising that any superparamagnetism for these contacts is observed at $4.2 \mathrm{~K}$, as the blocking temperature $T_{b}$ of the clusters, as determined from the magnetization of corresponding CIP samples ${ }^{27}$ are $180 \mathrm{~K}$ (type A) and $130 \mathrm{~K}$ (type B). Thus the clusters in the constriction are substantially smaller than intended. This follows from the expression for the blocking temperature, which is ${ }^{29}$

$$
T_{b}=\frac{K V}{25 k}
$$

Here $k$ is Boltzmann's constant, $K$ is the particle's anisotropy constant, and $V$ its volume. From Eq. (3) it follows that the cluster size, to give superparamagnetism at $4.2 \mathrm{~K}$, should be below $1 \mathrm{~nm}$ (effectively for our pancake-shaped granules $K$ $\approx 2 K_{s} / t_{\mathrm{gr}}=2.9 \times 10^{6} \mathrm{~J} / \mathrm{m}^{3}, K_{s}$ being the surface anisotropy constant of $\mathrm{Co} / \mathrm{Au}(111),{ }^{27}$ and $t_{\mathrm{gr}}$ being the granule thickness). To understand why the clusters are smaller than intended, we suggest that the nanohole in the membrane disturbs the Co growth in the constriction in comparison to the flat substrate situation of Ref. 27. The disturbance involves shadowing of the evaporation beam and deposition on the walls of the hole. These effects can give smaller clusters than intended.

On average, the GMR of type-B contacts exceeds that of type-A contacts. To explain this, we note that the higher growth rate for type $\mathrm{B}$ is expected to give a larger number of smaller Co clusters in the constriction region, in comparison to the clusters of type A. The clusters in type-B devices will therefore induce more interface scattering than those in type-A devices. Since interface scattering is most important for the GMR, type B will have the higher GMR, as observed. This observation agrees with CIP GMR data of granular bulk samples deposited in the same way. ${ }^{27}$ We note that, although on average $\mathrm{GMR}_{\text {type }}>\mathrm{GMR}_{\text {type A }}$, for individual pairs of contacts we also find $\mathrm{GMR}_{\text {type } \mathrm{B}}<\mathrm{GMR}_{\text {type A }}$ (see Table I). This results from the spread in the behavior. The spread and varying GMR nature for either contact type agree with the relatively low number of clusters expected, since the few clusters sampled by a point contact from a nonuniform size

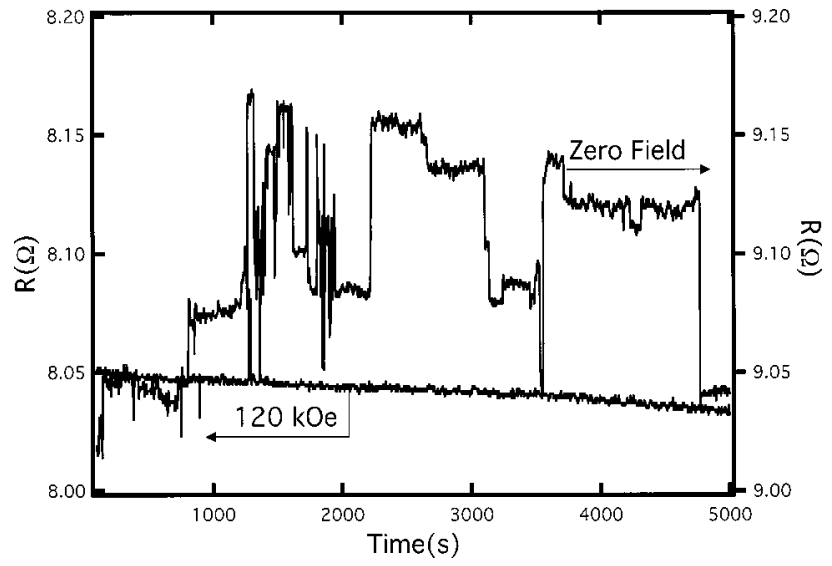

FIG. 10. Discrete fluctuations of the resistance of an 8.0- $\Omega$ granular $\mathrm{Co} / \mathrm{Au}$ point contact, measured at $H=0$ and $T=4.2 \mathrm{~K}$. At $H=120 \mathrm{kOe}$ the time trace is flat, as a result of alignment of the moments of the granules along the field direction.

distribution will have device-to-device fluctuations in their size and hence in the GMR. This effect will be enhanced by the variation in hole diameter, which gives different growth conditions for the Co clusters in the constriction.

Looking closely at the GMR curve of the $9.6-\Omega$ contact in Fig. 9(a), we notice steps or jumps. Such jumps were observed for the majority of the contacts. These sudden resistance changes are possibly due to reorientation of the magnetization of a single cluster. This would confirm that only a few Co clusters contribute to the GMR, as estimated and discussed above. Another indication of the involvement of only a few clusters is clear from Fig. 10, which shows discrete fluctuations in a time trace of the resistance of another contact, measured at $H=0$. In the trace at least five different discrete levels can be discerned. The occurrence of these levels is thought to correlate with different orientations of the magnetization of a few Co clusters. For an ensemble of clusters, i.e., a large system, the orientation fluctuations of the moments of the clusters, which correspond to fluctuations of the local strength of the spin dependent scattering, average out in the resistance, but for our small contacts the fluctuations survive. The absence of switching events and the low resistance state at $120 \mathrm{kOe}$ (see Fig. 10) corresponds to alignment of the moments of the clusters. In the field the moments have very little freedom, and, as for a multilayer with aligned magnetic layers, one spin channel undergoes weaker scattering and shorts the other channel, giving a low resistance.

Mostly, the point-contact spectra of the granular contacts are featureless, indicating diffusive transport. Occasionally, however, there are phonon peaks in the spectra. This is illustrated in Fig. 11 for a 16.2- $\Omega$ type-B contact (see also Table I). Clear peaks are present in the spectrum at $\pm 10 \mathrm{mV}$, the position of the transverse acoustic phonon peak of Au. Apparently in the $\mathrm{Co} / \mathrm{Au}$ contact the $\mathrm{Au}$ phonons are probed and not the Co phonons. This is not surprising in view of the $\mathrm{Au}$ volume fraction of $93 \%$. The clusters cause elastic scattering of electrons traversing the constriction. In view of the small number of clusters, the scattering will only be weak, inducing quasiballistic transport for some fraction of the electrons. Other electrons, for certain initial positions and velocity directions in the electrodes can follow a ballistic 


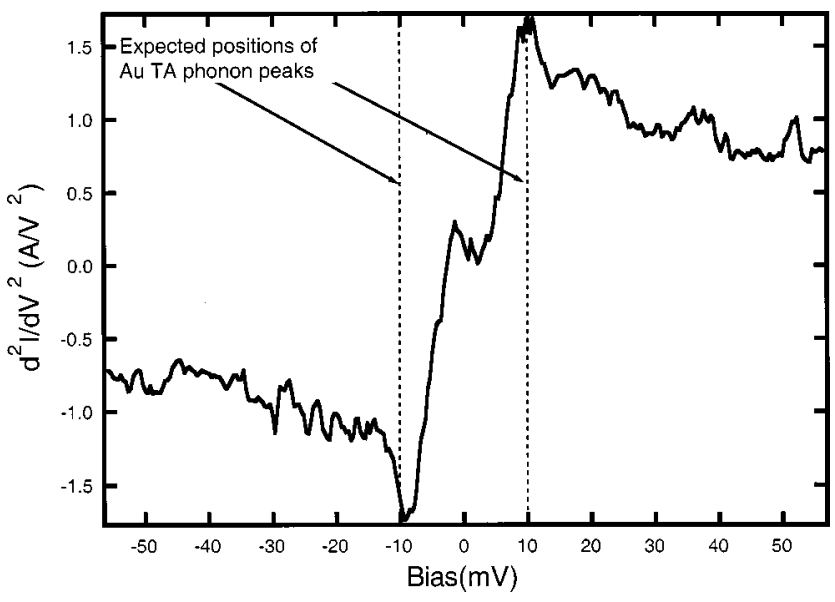

FIG. 11. Point-contact spectrum of a $16.2-\Omega$ granular $\mathrm{Co} / \mathrm{Au}$ point contact, measured at $T=4.2 \mathrm{~K}$. The spectrum has clear transverse acoustic (TA) phonon peaks at positions characteristic of Au. The GMR ratio of this contact is $11 \%$.

trajectory through the Au matrix in the constriction. Under an applied bias voltage, both for the ballistic and the quasiballistic trajectories a nonequilibrium electron distribution function is created, ${ }^{17,30}$ enabling emission of nonequilibrium phonons, corresponding to peaks in $d^{2} I / d V^{2}$. The chance to observe phonon peaks is higher for smaller contacts, since then it is easier to fulfill the criterion for (quasi-) ballistic transport. This transport is favored by fewer clusters in the constriction or a more favorable clusters arrangement in the constriction. These circumstances are part of the device-todevice variations, in accordance with the occasional observation of phonon peaks. In the spectrum of Fig. 11 longitudinal acoustic phonon peaks are absent and the background signal is high. This agrees with elastic scattering in the constriction. ${ }^{31}$ This demonstration of (quasi-)ballistic transport in a granular $\mathrm{Co} / \mathrm{Au}$ point contact opens the way to study the electron-energy dependence of the GMR effect.

\section{SUMMARY AND OUTLOOK}

We have fabricated and studied three generations of GMR point contacts. The first generation was made by deposition of a $\mathrm{Co} / \mathrm{Cu}$ multilayer after etching of the nanohole, while the second resulted from deposition of such a multilayer before etching of the nanohole. In both cases the sample was rotated ex situ to enable deposition of the counter electrode. Although the fabrication process of the second generation was designed to achieve an important improvement of the multilayer quality at the constriction, this did not lead to the expected increase of the GMR ratio, which was limited to $3 \%$ for the first and 5\% for the second generation. A possible explanation for this is the combined effect of contamination and a copper-oxide layer at the interface between the electrodes, both resulting from the ex situ rotation, and dead layers. In the third generation we applied in situ rotation of the sample and avoided the condition of not degrading or damaging the multilayer at the constriction by using granular $\mathrm{Co} / \mathrm{Au}$. The GMR ratio of the resulting granular contacts appeared strongly increased, to 14\%. GMR curves of the granular contacts exhibit characteristics of a granular system, in particular superparamagnetic behavior. From resistance jumps in the GMR curves and two-level fluctuations in time traces of the resistance it followed that only a few granules in the constriction are involved in generating the GMR effect. For one granular contact we observed phonon peaks in the point-contact spectrum, indicating ballistic transport.

The results give indications for improvements of the fabrication. In particular it is very tempting to develop a procedure of in situ etching of the nanohole and contacting of the multilayer through the hole, thus avoiding interfacial contamination and copper oxide. For this procedure contacts based on Si membranes are very attractive, since the final thin oxide of the membrane can be etched in the load lock of the deposition system, using gaseous HF. Another alternative is ex situ rotation combined with in situ surface cleaning with a low-energy argon beam. To avoid the dead-layer effect the insulating layer of the device should be deposited on top of the multilayer, which brings problems of its own (pinholes, adhesion). Spin valves, which have a spacer thickness beyond the range where coupling between the magnetic layers occurs, do not suffer from the dead-layer effect. Therefore a spin-valve point contact based on a Si membrane and hole etching in the load lock is an attractive possibility.

After submission of the manuscript we became aware of the results of Garcia, Munoz, and Zhao, ${ }^{32}$ who studied ballistic Ni-Ni nanocontacts. These contacts, made by bringing into contact two collinear sharpened $\mathrm{Ni}$ wires of which the magnetization was adjusted parallel or antiparallel, display a very strong MR when only a few quantum channels are transmitted. According to the authors this very interesting effect arises from a domain wall, which is centered at the constriction and which scatters electrons stronger when the constriction becomes smaller. This effect cannot play a role in our multilayer contacts. The reason is that the structure of our contacts is completely different from that of the $\mathrm{Ni}-\mathrm{Ni}$ contacts, so that a domain wall cannot be present in the constriction. Moreover, from our results on homogeneous Co, $\mathrm{Ni}$, and permalloy point contacts, ${ }^{33}$ which have the same geometry as the present multilayer contacts, we know that the specific MR of Ref. 32 does not occur for resistances in the range $1-20 \Omega$.

\section{ACKNOWLEDGMENTS}

We acknowledge support by EPSRC, NWO, and the British Council (Grant No JRP 370). This work is part of the research program of the "Stichting Fundamenteel Onderzoek der Materie (FOM),', which is financially supported by the "Nederlandse Organisatie voor Wetenschappelijk Onderzoek (NWO).' We thank G. E. W. Bauer and R. Coehoorn for stimulating discussions.
*Permanent address: B. I. Verkin Institute of Low Temperature Physics and Engineering, Academy of Science of Ukraine, 47 Lenin Avenue, Kharkiv 310164, Ukraine.

${ }^{1}$ M. N. Baibich, J. M. Broto, A. Fert, F. Nguyen, F. Petroff, P.
Etienne, G. Creuzet, A. Friederich, and J. Chazelas, Phys. Rev. Lett. 61, 2472 (1988).

${ }^{2}$ R. Schad, C. D. Potter, P. Belien, G. Verbanck, V. V. Moshchalkov, and Y. Bruynseraede, Appl. Phys. Lett. 64, 3500 
(1994)

${ }^{3}$ W. P. Pratt, Jr., S.-F. Lee, J. M. Slaughter, R. Loloee, P. A. Schroder, and J. Bass, Phys. Rev. Lett. 66, 3060 (1991). In later experiments these authors used $\mathrm{NbTi}$ contact strips, so that the magnetic-field range was extended to $3.5 \mathrm{~T}$.

${ }^{4}$ T. Valet and A. Fert, Phys. Rev. B 48, 7099 (1993).

${ }^{5}$ M. A. M. Gijs, S. K. J. Lenczowski, and J. B. Giesbers, Phys. Rev. Lett. 70, 3343 (1993).

${ }^{6}$ L. Piraux, J. M. George, J. F. Despres, C. Leroy, E. Ferain, R. Legras, K. Ounadjela, and A. Fert, Appl. Phys. Lett. 65, 2428 (1994).

${ }^{7}$ A. Blondel, J. P. Meier, B. Doudin, and J. Ph. Ansermet, Appl. Phys. Lett. 65, 3019 (1994).

${ }^{8}$ W. P. Pratt, Jr., S.-F. Lee, P. Holody, Q. Yang, R. Loloee, J. Bass, and P. A. Schroder, J. Magn. Magn. Mater. 126, 406 (1993).

${ }^{9}$ K. M. Schep, P. J. Kelly, and G. E. W. Bauer, Phys. Rev. Lett. 74, 586 (1995)

${ }^{10}$ G. E. W. Bauer, Phys. Rev. B 51, 16984 (1995).

${ }^{11}$ M. V. Tsoi, A. G. M. Jansen, and J. Bass, J. Appl. Phys. 81, 5530 (1997).

${ }^{12}$ P. A. M. Holweg, J. Caro, A. H. Verbruggen, and S. Radelaar, Microelectron. Eng. 11, 27 (1990); N. N. Gribov, J. Caro, and S. Radelaar, Physica B 97, 218 (1997).

${ }^{13}$ N. N. Gribov, S. J. H. C. Theeeuwen, J. Caro, and S. Radelaar, Microelectron. Eng. 35, 317 (1997); N. N. Gribov, S. J. C. H. Theeuwen, J. Caro, E. van der Drift, F. D. Tichelaar, T. R. de Kruijff, and B. J. Hickey, J. Vac. Sci. Technol. B 16, 3943 (1998).

${ }^{14}$ S. S. P. Parkin, in Ultrathin Magentic Structures II, edited by B. Heinrich and J. A. C. Bland (Springer, New York, 1994), p. 173.

${ }^{15}$ T. C. McGuire and I. R. Potter, IEEE Trans. Magn. 10, 1018 (1975).

${ }^{16}$ W. Schwarzacher and D. S. Lashmore, IEEE Trans. Magn. 32, 3133 (1996); W. Schwarzacher (private communication).

${ }^{17}$ I. K. Yanson, Fiz. Nizk. Temp, 9, 676 (1983) [Sov. J. Low Temp. Phys. 9, 343 (1983)].
${ }^{18}$ J. C. Maxwell, A Treatise on Electricity and Magnetism (Dover, New York, 1954).

${ }^{19}$ M. A. M. Gijs, J. B. Giesbers, S. K. J. Lenczowski, and H. H. J. M. Janssen, Appl. Phys. Lett. 63, 111 (1993).

${ }^{20}$ ANSYS 5.3, Swanson Analysis Systems, Inc., Houston, PA 153420065, USA.

${ }^{21}$ H. M. Swartjes, A. G. M. Jansen, and P. Wyder, Phys. Rev. B 38, 8114 (1988).

${ }^{22}$ The differences with our result arise from the omission of the $\varphi$ dependence in Ref. 11 and a too weak reduction of $R_{e l}$ by the $\varphi$-dependent term in Ref. 21. Another difference is that in Refs. 11 and 21 the identification $D^{*}=4 t$ is made, which partially counteracts a too weak $\varphi$-dependent term or its absence.

${ }^{23}$ B. J. Hickey, K. P. Wellock, and M. J. Walker, J. Appl. Phys. 81, 4476 (1997).

${ }^{24}$ L. Tang and D. E. Laughlin, J. Appl. Phys. 81, 4906 (1997).

${ }^{25}$ P. K. Krishnamoorthy and S. C. Sircar, Oxid. Met. 2, 349 (1970).

${ }^{26}$ C. L. Chien, J. Q. Xiao, and J. S. Jiang, J. Appl. Phys. 73, 5309 (1993).

${ }^{27}$ J. Xu, M. A. Howson, E. Kolb, P. Veillet, and A. Pelford-Long, J. Magn. Magn. Mater. 192, 35 (1999).

${ }^{28}$ To be consistent with the multilayer contacts, we define the GMR ratio for the granular contacts as $[R(0)-R(120 \mathrm{kOe})] /$ $R(120 \mathrm{kOe})$, although for granular systems an alternative definition is sometimes preferred.

${ }^{29}$ I. S. Jacobs and C. P. Bean, in Magnetism III, edited by G. T. Rado and H. Suhl (Academic, New York, 1963), p. 271.

${ }^{30}$ I. O. Kulik, R. I. Shekhter, and A. G. Shkorbatov, Zh. Eḱsp. Teor. Fiz. 81, 2126 (1981) [Sov. Phys. JETP 54, 1130 (1981)].

${ }^{31}$ I. K. Yanson, I. O. Kulik, and A. G. Batrak, J. Low Temp. Phys. 42, 527 (1981).

${ }^{32}$ N. Garcia, M. Munoz, and Y.-W. Zhao, Phys. Rev. Lett. 82, 2923 (1999).

${ }^{33}$ R. P. van Gorkom, J. Caro, S. J. H. C. Theeuwen, K. P. Wellock, N. N. Gribov, and S. Radelaar, Appl. Phys. Lett. 74, 422 (1999). 